\section{Confiabilidade da informação sobre hospitalizações por causas externas de um hospital público em São José dos Campos, São Paulo, Brasil}

\author{
Data reliability of hospital \\ admissions due to external causes \\ in a public hospital in São José dos \\ Campos, São Paulo, Brazil
}

Luís Paulo Rodrigues Melione' Maria Helena Prado de Mello Jorge ${ }^{2}$

'Secretaria Municipal de Saúde. Prefeitura Municipal de São José dos Campos. ${ }^{2}$ Departamento de Epidemiologia. Faculdade de Saúde Pública. Universidade de São Paulo, São Paulo.

Baseado na Dissertação de Mestrado do autor principal "Morbidade hospitalar por causas externas no Sistema Único de Saúde em São José dos Campos, SP", apresentada à Faculdade de Saúde Pública da USP, em 2006

Correspondência: Luís Paulo Rodrigues Melione. Rua José de Alencar, 123, 5ª andar, sala 1 - São José dos Campos, SP - CEP 12209-530. E-mail: Imelione@uol.com.br

\section{Resumo}

Objetivo: Conhecer a qualidade dos dados de internação por causas externas em São José dos Campos, São Paulo. Método: Foram estudadas as internações pelo Sistema Único de Saúde por lesões decorrentes de causas externas no primeiro semestre de 2003, no Hospital Municipal, referência para o atendimento ao trauma no Município, por meio da comparação dos dados registrados no Sistema de Informações Hospitalares com os prontuários de 990 internações. A concordância das variáveis relativas à vítima, à internação e ao agravo foi avaliada pela taxa bruta de concordância e pelo coeficiente Kappa. As lesões e as causas externas foram codificadas segundo a $10^{\text {a }}$ revisão da Classificação Internacional de Doenças, respectivamente, capítulos XIX e XX. Resultados: A taxa de concordância bruta foi de boa qualidade para as variáveis relativas à vítima e à internação, variando de $89,0 \%$ a $99,2 \%$. As lesões tiveram concordância ótima, exceto os traumatismos do pescoço ( $\mathrm{k}=0,73)$, traumatismos múltiplos $(\mathrm{k}=0,67)$ e fraturas do tórax $(\mathrm{k}=0,49)$. As causas externas tiveram concordância ótima para acidentes de transporte $(\mathrm{k}=0,90)$ e quedas $(\mathrm{k}=0,83)$. A confiabilidade foi menor para agressões $(\mathrm{k}=0,50)$, causas indeterminadas ( $\mathrm{k}=0,37)$, e complicações da assistência médica $(k=0,03)$. Houve concordância ótima nos acidentes de transporte em pedestres, ciclistas e motociclistas. Conclusão: A maioria das variáveis de estudo teve boa qualidade no nível de agregação analisado. Algumas variáveis relativas à vítima e alguns tipos de causas externas necessitam de aperfeiçoamento da qualidade dos dados. $\mathrm{O}$ perfil da morbidade hospitalar encontrado confirmou os acidentes de transporte como importante causa externa de internação hospitalar no Município.

Palavras chave: Acidentes. Causas Externas. Confiabilidade. Hospitalização. Lesões. Morbidade. Sistemas de Informação. Violência. 


\section{Abstract}

Objective: To identify the quality of externalcause hospital morbidity admission data in the city of São José dos Campos, São Paulo, Brazil. Method: Admissions via the Unified Health System to the Municipal Hospital, a reference trauma care hospital in the city, resulting from external-cause injuries were analyzed during the first semester of 2003, by comparing the data entered in the Hospital Information System (SIH) to 990 patient admission charts. Agreement between variables regarding victim, admission, and the offence was analyzed by rate of gross agreement and by the Kappa coefficient. Injuries and external causes were coded according to the International Classification of Diseases, $10^{\text {th }}$ revision, chapters XIX and XX respectively. Results: The rate of crude agreement was very good for variables regarding victim and admission with an $89.0 \%$ to $99.2 \%$ variation. Injuries had very good agreement, except for neck traumas ( $\mathrm{k}=0.73)$, multiple trauma $(\mathrm{k}=0.50)$, and chest fractures $(\mathrm{k}=0.49)$. External causes had very good agreement for land transportation accidents $(\mathrm{k}=0.90)$ and falls $(\mathrm{k}=0.83)$. Reliability was smaller for assaults $(\mathrm{k}=0.50)$, undetermined intent events $(\mathrm{k}=0.37)$, and medical care complications $(\mathrm{k}=0.03)$. There was very good agreement for transportation accidents involving pedestrians, cyclists, and motorcyclists. Conclusion: Most of the variables in the study were good in the aggregation level analyzed. Some variables regarding the victim and some types of external cause require data quality improvement. The profile of hospital morbidity found in this study has confirmed that land transportation accidents are an important external cause for admission in the city.

Keywords: Accidents. External Causes. Hospitalization. Information Systems. Injuries. Morbidity. Reliability. Violence.

\section{Introdução}

O conhecimento do perfil epidemiológico das causas externas e suas vítimas tem sido adquirido, principalmente, utilizandose dados de mortalidade, pois são obtidos mais facilmente, de forma sistematizada e com melhor qualidade ${ }^{1}$. Por se tratar de evento único e atingir todos os estratos sociais, o óbito é importante fonte de informação para avaliar a situação de saúde de uma população. Entretanto, algumas vezes tem sido encontrada baixa concordância entre a causa básica registrada na Declaração de Óbito e o tipo de causa externa conhecida após investigação ${ }^{2}$. Estima-se que, nos países desenvolvidos, para cada óbito por lesões, 30 vítimas sejam hospitalizadas e 300 tratadas em serviços de emergência e depois liberadas ${ }^{3}$, o que mostra a importância de se dispor de dados de morbidade.

Neste contexto, tornaram-se necessários sistemas de informação, confiáveis, também sobre a morbidade por acidentes e violências e que pudessem captar as vítimas sobreviventes no momento do agravo. Os dados sobre morbidade por causas externas são escassos e de difícil análise, tanto pelo subregistro dos eventos como pela falta de sistemas de informação específicos sobre esse tema. Geralmente são aproveitadas bases de dados originalmente desenvolvidas para o faturamento de internações hospitalares, como é o caso do Sistema de Informações Hospitalares do Sistema Único de Saúde (SIH-SUS), que tem como unidade de registro a Autorização de Internação Hospitalar (AIH). Entretanto, existem limitações para o uso dos dados sobre diagnósticos médicos colhidos com finalidade de pagamento. A codificação de doenças é feita por médicos que atenderam o paciente ou por pessoal administrativo, ambos não preparados para a função de codificação de diagnósticos ${ }^{4}$. No entanto, o SIH-SUS é um sistema ágil, que disponibiliza informações identificadas aos gestores locais, com defasagem inferior a um mês, e que pode ser utilizado como fonte de informação para vigilância epidemiológica e avaliação e controle de ações de saúde ${ }^{5}$. 
Existem poucos estudos sobre a confiabilidade dos dados de internação hospitalar ${ }^{6}$. Lebrão ${ }^{7}$ realizou análise de fidedignidade dos dados estatísticos hospitalares, anterior à existência da $\mathrm{AIH}$, tendo verificado maior concordância nas variáveis demográficas e administrativas e menor concordância na variável diagnóstico. Veras e Martins ${ }^{8}$ estudaram a confiabilidade dos dados das AIHs e também encontraram piores resultados para variáveis clínicas do que para as variáveis demográficas e administrativas. Mathias e Soboll ${ }^{9}$ avaliaram a qualidade dos dados do campo diagnóstico principal das AIHs tendo encontrado maiores concordâncias em níveis de maior agregação dos diagnósticos. Outro estudo, realizado com a finalidade de avaliar a qualidade das informações sobre infarto agudo do miocárdio no SIH-SUS ${ }^{10}$, identificou resultados satisfatórios. O estudo de Andrade e Mello Jorge ${ }^{11}$ encontrou uma concordância regular nas internações por acidentes de transporte. Já Tomimatsu ${ }^{12}$ estudou as internações por causas externas no SIH-SUS e identificou problemas na qualidade dos dados sobre internações por quedas e causas indeterminadas.

A implantação da décima revisão da Classificação Internacional de Doenças (CID-10) ${ }^{13}$ para morbidade no SIH-SUS ${ }^{14} \mathrm{e}$ a obrigatoriedade do registro nesse sistema, no campo "diagnóstico principal" do código referente à natureza da lesão e no campo "diagnóstico secundário", a causa externa que a motivou ${ }^{15}$, representaram avanços na compreensão da epidemiologia das internações hospitalares por causas externas.

O objetivo deste estudo foi analisar a concordância da codificação dos diagnósticos principal e secundário e de variáveis relativas à vítima e à internação, entre a base de dados do SIH-SUS e o prontuário do paciente.

\section{Material e método}

O Município de São José dos Campos fica situado no Vale do Paraíba, região leste do Estado de São Paulo, com uma população estimada para 2003 de 569.172 habitantes ${ }^{16}$. Possui intenso tráfego urbano e regional, sendo cortado pela Rodovia Presidente Dutra.

O material de estudo foi constituído das internações por causas externas pagas pelo SUS no Hospital Municipal Dr. José de Carvalho Florence, em São José dos Campos, SP, entre 01 de janeiro e 30 de junho de 2003. Esse hospital é considerado referência para trauma e as internações ali realizadas representaram $92,3 \%$ do total de internações por lesões decorrentes de causas externas pelo SUS no Município no mesmo período. As internações financiadas pelo Sistema Único de Saúde foram responsáveis por $81,0 \%$ do total de internações ocorridas na Região de Saúde de São José dos Campos, em $2002^{17}$.

\section{Fontes de dados}

- Base de dados de AIHs, pagas pelo Ministério da Saúde. Essa base de dados foi obtida dos arquivos públicos disponibilizados em meio eletrônico pelo Departamento de Informática do SUS (DATASUS) ${ }^{18}$. As bases de dados do ano de competência 2003 (janeiro a dezembro) foram consultadas para a seleção das internações com data de entrada entre primeiro de janeiro e trinta de junho de 2003.

- Base de dados de AIHs identificadas (com nome, endereço e número do prontuário do paciente) e apresentadas pelo Município. Os dados foram obtidos do programa SISAIH01 - Sistema de AIH em disquete, desenvolvido pelo DATASUS, disponível no Município. Os critérios para consultas as bases de dados foram os mesmos já citados no item anterior.

- Prontuários médicos dos pacientes internados por causas externas. Os números dos prontuários foram obtidos do banco de dados de AIHs identificadas do Município. Esses prontuários estavam arquivados no Hospital Municipal. Foram encontrados 990 prontuários das 993 AIHs de pacientes internados por causas externas por esse método. A 
consulta foi registrada num formulário desenvolvido para a pesquisa.

Foram selecionadas todas as internações de pacientes residentes e não residentes, com ocorrência ou não no Município, que contivessem, independentemente, o diagnóstico principal no capítulo XIX (Lesões, envenenamentos e algumas outras conseqüências de causas externas) da CID10, ou no capítulo XX (Causas externas de morbidade e de mortalidade); o diagnóstico secundário no capítulo XIX ou no capítulo $\mathrm{XX}$. Este procedimento visou recuperar registros que, eventualmente, tivessem sido codificados em desacordo com as regras da CID- $10^{13}$ e do próprio SIH-SUS ${ }^{15}$.

Inicialmente, foi realizada a procura de internações por lesões provocadas por acidentes e violências nas bases de dados das AIHs pagas, localizadas e selecionadas pelos critérios já informados. Posteriormente, foi realizada a mesma busca para as AIHs identificadas. Desta forma, vincularam-se todas as internações pagas, em banco de dados não identificados, com as AIHs identificadas apresentadas pelo Município.

Foram verificados, no estudo, os mesmos documentos existentes, à época, nos prontuários dos pacientes e passíveis de consulta pelos funcionários do setor de faturamento do hospital, quando da codificação original. Os diagnósticos (principal e secundário) foram codificados a posteriori, pelo pesquisador, sem o conhecimento da codificação no SIH-SUS. O processo de codificação da lesão e da causa externa seguiu as regras de codificação de morbidade por causa única preconizado pela CID-10. O pesquisador foi qualificado como multiplicador no uso da CID-10 pelo Centro Brasileiro de Classificação de Doenças, vinculado por convênio à Organização Pan-americana da Saúde, ao Ministério da Saúde e à Universidade de São Paulo.

As bases de dados de AIHs pagas e AIHs identificadas foram relacionadas e transformadas em um único banco de dados, para facilitar a identificação do número do prontuário e agilizar a emissão do formulário de pesquisa. A chave primária de relacionamento dos bancos de dados de internação hospitalar foi o número da AIH, o mês e o ano de competência. Desta forma, as AIHs reapresentadas no período foram consideradas, no lugar daquelas recusadas quando da sua primeira apresentação. As variáveis foram codificadas e foram relacionados os bancos de dados de AIH e prontuários (avaliação do pesquisador).

As variáveis de estudo foram àquelas relativas à vítima, a internação e ao agravo. As relativas à vítima foram: sexo, data de nascimento e idade (esta foi calculada a partir da diferença entre a data de nascimento e a data de internação); local de residência: logradouro, bairro e município de residência. $\mathrm{O}$ bairro de residência somente pode ser avaliado no SIH-SUS em São José dos Campos porque foi implantada, desde 1998, uma tabela de bairros no sistema ${ }^{19}$. Essa a codificação foi introduzida na rotina de digitação dos arquivos de AIHs apresentadas (programa SISAIH01-Sistema de AIH em disquete), desenvolvida pelo DATASUS. Quanto ao município de residência existem duas variáveis no banco de dados, sendo a primeira registrada em campo descritivo e o segunda em campo com o código IBGE do município.

As variáveis relativas à internação foram: data de internação, data de alta, tempo de permanência (em dias, medido a partir da diferença entre a data de internação e a data de alta hospitalar) e tipo de saída hospitalar (alta ou óbito). A variável "procedimento realizado" foi levada em consideração apenas para a codificação do diagnóstico principal pelo pesquisador na avaliação do prontuário. Não houve o objetivo de avaliar sua qualidade.

As variáveis relativas ao agravo foram: diagnóstico principal e secundário. $\mathrm{O}$ diagnóstico principal é definido como a afecção primariamente responsável pela necessidade de tratamento ou investigação do paciente. Foram selecionados os códigos S00 a T98 da CID-10 (Capítulo XIX), sendo constituídos dois grupos de análise: segmento corporal afetado e natureza da 
lesão. Segmento corporal afetado: S00 a S09 (cabeça), S10 a S19 (pescoço), S20 a S29 (tórax), S30 a S39 (abdome, dorso e pelve), S40 a S69 (membros superiores), S70 a S99 (membros inferiores) e T00 a T07 (traumatismos múltiplos). Natureza da lesão: S02 (fratura crânio e ossos da face), S12 (fratura do pescoço), S22 (fratura do tórax: costelas, esterno e coluna torácica), S32 (fratura de coluna lombar e da pelve), S42 (fratura do ombro e do braço), S52 (fratura do antebraço), S62 (fratura do punho e da mão), S72 (fratura do fêmur), S82 (fratura da perna, incluindo tornozelo) e S92 (fratura do pé); S03, S13, S23, S33, S43, S53, S63, S73, S83, S93 e T03 (luxações, entorses e distensões), S06 (traumatismo intracraniano), S27 (traumatismo de outros órgãos intratorácicos), S36 (traumatismo de órgãos intra-abdominais), T01 a T07 (traumatismos múltiplos), T20 a T32 (queimaduras e corrosões), T36 a T65 (intoxicações), T79 a T88 (complicações de cuidados médicos e cirúrgicos), T90 a T98 (seqüelas de causas externas); todas as demais categorias do capítulo XIX (demais lesões). A avaliação de concordância para o diagnóstico principal foi realizada para os níveis de quatro caracteres, de três caracteres e agrupamentos da CID-10.

O diagnóstico secundário foi obrigatoriamente uma causa externa, desde que o diagnóstico principal tivesse sido uma lesão, segundo regra internacional de codificação da CID- $10^{13}$ e do SIH-SUS ${ }^{15}$. Foram selecionados os códigos de categorias V01 a Y98 (Capítulo XX) e foram constituídos os seguintes grupos: V01 a V99 (acidentes de transporte), W01 a W19 (quedas), X85 a Y09 e Y35 a Y36 (agressões), X60 a X84 (lesões autoprovocadas intencionalmente), Y40 a Y84 (complicações de assistência médica e cirúrgica), Y10 a Y34 (eventos cuja intenção é indeterminada), Y85 a Y89 (seqüelas de causas externas); todas as demais categorias do capítulo XX (demais causas acidentais).

Dentro dos agrupamentos: acidentes de transporte, quedas e causas indeterminadas, alguns subgrupos mais freqüentes foram analisados, entre eles, pedestres (V01 a V09), ciclistas (V10 a V19), motoci- clistas (V20 a V29), ocupante de automóvel e caminhonete (V40 a V59), queda não especificada (W17 a W19) e eventos e intenção indeterminados (Y34). A avaliação de concordância para o diagnóstico secundário também foi realizada para os níveis de quatro caracteres, de três caracteres e agrupamentos da CID-10.

Foram utilizadas: a taxa de concordância bruta e o coeficiente Kappa ${ }^{20}$ para a análise de concordância das variáveis de estudo. A taxa de concordância bruta mede a proporção de resultados concordantes em variáveis expressas em categorias, mas não leva em consideração a concordância devida ao acaso. Foi o método utilizado para análise da maioria das variáveis. As menores concordâncias esperadas eram para as variáveis relativas ao agravo: diagnósticos principal e secundário da AIH, codificados em CID-10 em comparação com os diagnósticos identificados nos prontuários dos pacientes.

Houve grande variação de concordância no campo diagnóstico secundário segundo diferentes agrupamentos da CID-10. Por essa razão, os agrupamentos de lesões segundo segmento corporal afetado, natureza da lesão e de causas externas (por tipo de causa e qualidade da vítima) foram analisados com o auxílio do coeficiente Kappa ${ }^{20}$. Esse coeficiente leva em consideração a concordância devido ao acaso, que é descontada no resultado final. Trabalhou-se com um intervalo de confiança de $95 \%$.

Para a interpretação do coeficiente Kappa foi utilizada a classificação de Landis Jr e $\mathrm{Koch}^{21}$ adaptada por Pereira ${ }^{22}$. Nesta interpretação considera-se: concordância ruim um valor de Kappa $<0,00$; concordância fraca um valor entre 0,00 e 0,20 ; concordância sofrível entre 0,21 e 0,40 ; regular entre 0,41 e 0,60 ; boa entre 0,61 e 0,80 ; ótima entre 0,81 e 0,99 e concordância perfeita um valor igual a 1,00 .

Para as tabulações de número, proporção e taxa de concordância bruta dos dados importados, oriundos do SIH-SUS, e dos dados originais coletados de prontuários, foi utilizado o programa de domínio público EPI-INFO ${ }^{23}$. Para a análise de concordância, 
realizada através do coeficiente Kappa ${ }^{20}$, foi utilizado o programa de domínio público PEPI 4.0, desenvolvido por Abramson e Gahlinger ${ }^{24}$.

O projeto de pesquisa foi aprovado pelo Comitê de Ética e Pesquisa da Faculdade de Saúde Pública da Universidade de São Paulo em 23 de março de 2005, Protocolo de Pesquisa no 1.230 .

\section{Resultados}

Foram encontrados 990 prontuários das 993 AIHs de pacientes internados por causas externas $(99,7 \%)$. Houve, entretanto, dificuldades na consulta aos prontuários, tais como: letra pouco legível, prontuários sem informação sobre a causa externa de lesão e mais de um documento com informações diferentes sobre a causa externa na mesma internação. As palavras pouco legíveis foram todas compreendidas. A maior dificuldade para o pesquisador foi a alta proporção de registros incompletos sobre as causas externas, o que comprometeu a qualidade da informação. Tanto em relação à lesão como a causa externa, optou-se por selecionar a informação mais específica que pudesse ser obtida.

\section{Variáveis relativas à vítima}

A taxa de concordância bruta para sexo foi de $98,9 \%$, para idade $(93,0 \%)$, data de internação $(99,2 \%)$ e data de nascimento $(91,0 \%)$, embora esta última tenha sido a maior responsável pelas diferenças encontradas na idade. A taxa de concordância bruta para a variável município (descrição) foi de 90,7\% e para município (código IBGE), 91,1\% (Tabela 1)

A concordância foi boa em ambos os casos da variável município, porém a proporção de internações de residentes em São José dos Campos, segundo o código do Município no banco de dados, era de 99,7\% e segundo a consulta ao prontuário foi de 89,4\% (Tabela 2).

A colocação do código IBGE do município de residência é obrigatória no SIH-SUS.
Nos casos de município de residência "ignorado”, foi digitado, no hospital, o código IBGE de São José dos Campos (16 casos). Na situação de outro local de residência (89 casos), somente 3 foram digitados com o código do respectivo município. Nos demais 86 casos, foi digitado o código de São José dos Campos

A taxa de concordância bruta da variável bairro de residência foi de $89,0 \%$ (Tabela 1 ). A variável "logradouro" somente está disponível no banco de dados de AIH identificadas no nível municipal. Foi analisada por ter importância para a correta identificação de bairro de residência. A taxa de concordância bruta foi de $91,7 \%$.

\section{Variáveis relativas à internação}

As variáveis de internação utilizadas a partir do banco de dados da AIH foram: número do prontuário, tempo de permanência e tipo de saída hospitalar. O número do prontuário estava correto no banco de dados identificado do SIH-SUS em 98,3\% dos casos, o que facilitou a localização dos mesmos para a pesquisa de campo. O tempo de permanência teve taxa de concordância bruta de 98,7\%, a data de internação, 99,2\% e a data de alta, 99,4\% (Tabela 1). A variável: tipo de saída hospitalar teve uma confiabilidade alta, com taxa de concordância bruta de $99,0 \%$.

\section{Variáveis relativas ao agravo}

A concordância bruta para a variável diagnóstico principal foi de $54,9 \%$ em nível de quatro caracteres da CID-10, de 84,9\% em nível de três caracteres e 90,4\% em agrupamentos (Tabela 1).

Os resultados obtidos para a variável diagnóstico secundário foram os piores nessa avaliação. Em nível de quatro caracteres, a concordância foi de $43,8 \%$, para três caracteres, subiu para $50,1 \%$, e para agrupamentos chegou a $66,9 \%$ (Tabela 1 ).

Os resultados obtidos com o cruzamento das respostas concordantes e discordantes entre o SIH-SUS e a avaliação do 
Tabela 1 - Taxa de concordância bruta de variáveis relativas às internações por causas externas, no SIH e na avaliação do pesquisador, no Hospital Municipal de São José dos Campos, primeiro semestre de 2003 ( $n=990$ ).

Table 1 - Rate of crude agreement of variables related to hospital admissions due to external causes, in the SIH and in the

\begin{tabular}{|c|c|c|c|}
\hline varegreper's evgluation, in the Municipal Hospita & $\begin{array}{l}\text { רo łpsédos Camp } \\
\text { concordâncias }\end{array}$ & 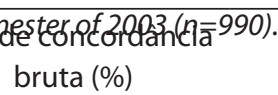 & IC 95\% \\
\hline \multicolumn{4}{|l|}{ Relativas à vítima } \\
\hline Sexo & 979 & 98,9 & $98,0-99,4$ \\
\hline Idade (em anos) & 921 & 93,0 & $91,2-94,5$ \\
\hline Data de nascimento & 901 & 91,0 & $89,0-92,7$ \\
\hline Município de residência (descrição) & 898 & 90,7 & $88,7-92,4$ \\
\hline Município de residência (código IBGE) & 902 & 91,1 & $89,1-92,8$ \\
\hline Bairro de residência & 881 & 89,0 & $86,8-90,8$ \\
\hline Logradouro & 908 & 91,7 & $89,8-93,3$ \\
\hline \multicolumn{4}{|l|}{ Relativas à internação } \\
\hline Número do prontuário & 973 & 98,3 & $97,2-99,0$ \\
\hline Data de internação & 982 & 99,2 & $98,3-99,6$ \\
\hline Data de alta & 984 & 99,4 & $98,6-99,8$ \\
\hline Tempo de permanência (em dias) & 977 & 98,7 & $97,7-99,3$ \\
\hline Tipo de saída hospitalar & 980 & 99,0 & $98,1-99,5$ \\
\hline \multicolumn{4}{|l|}{ Relativas ao agravo } \\
\hline Diagnóstico principal (4 caracteres)* & 544 & 54,9 & $51,8-58,1$ \\
\hline Diagnóstico principal (3 caracteres)* & 841 & 84,9 & $82,5-87,1$ \\
\hline Diagnóstico principal (agrupamentos)* & 895 & 90,4 & $88,4-92,1$ \\
\hline Diagnóstico secundário (4 caracteres)* & 434 & 43,8 & $40,7-47,0$ \\
\hline Diagnóstico secundário (3 caracteres)* & 496 & 50,1 & $46,9-53,3$ \\
\hline Diagnóstico secundário (agrupamentos)* & 662 & 66,9 & $63,8-69,8$ \\
\hline
\end{tabular}

* Classificação Internacional de Doenças, décima revisão (CID-10)/*International Classification of Diseases, $10^{\text {th }}$ revision.

Tabela 2 - Comparação do município de residência pelo código IBGE no SIH e na avaliação do pesquisador, de internações por causas externas, no Hospital Municipal de São José dos Campos, primeiro semestre de 2003.

Table 2 - Comparison of the city of residence by IBGE code in the SIH and in the researcher's evaluation, of admissions due to external causes, in the Municipal Hospital of São José dos Campos, first semester of 2003.

\begin{tabular}{|c|c|c|c|c|}
\hline \multirow[t]{2}{*}{ Município de residência } & \multicolumn{2}{|c|}{$\mathrm{SIH}$} & \multicolumn{2}{|c|}{ Avaliação do pesquisador } \\
\hline & $\mathrm{N}$ & $\%$ & $\mathrm{~N}$ & $\%$ \\
\hline São José dos Campos & 987 & 99,7 & 885 & 89,4 \\
\hline Municípios da Regional de Saúde* & 3 & 0,3 & 67 & 6,8 \\
\hline Outros Municípios de SP & - & - & 19 & 1,9 \\
\hline Municípios de outros Estados & - & - & 3 & 0,3 \\
\hline Ignorado & - & - & 16 & 1,6 \\
\hline Total & 990 & 100,0 & 990 & 100,0 \\
\hline
\end{tabular}


Tabela 3 - Concordância entre o SIH e a avaliação do pesquisador, das variáveis relativas ao agravo, através do coeficiente Kappa, de internações por causas externas, no Hospital Municipal de São José dos Campos, primeiro semestre de 2003 ( $n=990)$.

Table 3 - Agreement between the SIH and the researcher's evaluation of the variables related to the offence by means of Kappa coefficient of admissions due to external causes in the Municipal Hospital of São José dos Campos, first semester of 2003 $(n=990)$.

\begin{tabular}{|c|c|c|c|}
\hline \multirow[t]{2}{*}{ Agrupamentos da CID-10 } & \multirow{2}{*}{$\begin{array}{c}\text { Número de } \\
\text { concordâncias }\end{array}$} & \multicolumn{2}{|c|}{ Kappa } \\
\hline & & Valor & IC 95\% \\
\hline \multicolumn{4}{|l|}{ Segmento corporal afetado } \\
\hline Traumatismos da cabeça & 969 & 0,94 & $0,91-0,96$ \\
\hline Traumatismos do pescoço & 982 & 0,73 & $0,55-0,91$ \\
\hline Traumatismos do tórax & 980 & 0,83 & $0,72-0,93$ \\
\hline Traumatismos do abdome e pelve & 977 & 0,93 & $0,89-0,97$ \\
\hline Traumatismos de membro superior & 970 & 0,94 & $0,91-0,97$ \\
\hline Traumatismos de membro inferior & 971 & 0,95 & $0,93-0,97$ \\
\hline Traumatismos múltiplos & 964 & 0,67 & $0,55-0,79$ \\
\hline \multicolumn{4}{|l|}{ Natureza da lesão } \\
\hline Fraturas & 936 & 0,89 & $0,86-0,92$ \\
\hline Fratura do crânio e dos ossos da face & 988 & 0,92 & $0,87-0,97$ \\
\hline Fratura do pescoço & 984 & 0,72 & $0,51-0,94$ \\
\hline Fratura de tórax & 980 & 0,49 & $0,22-0,77$ \\
\hline Fratura da coluna lombar e pelve & 981 & 0,88 & $0,79-0,96$ \\
\hline Fratura do ombro e do braço & 984 & 0,91 & $0,84-0,98$ \\
\hline Fratura do antebraço & 985 & 0,97 & $0,94-1,00$ \\
\hline Fratura do punho e da mão & 982 & 0,90 & $0,83-0,97$ \\
\hline Fratura do fêmur & 984 & 0,97 & $0,95-0,99$ \\
\hline Fratura da perna & 981 & 0,95 & $0,92-0,98$ \\
\hline Fratura do pé & 990 & & \\
\hline Luxações, entorses e distensões & 979 & 0,82 & $0,72-0,92$ \\
\hline Traumatismo intracraniano & 978 & 0,95 & $0,91-0,98$ \\
\hline Traumatismo do pulmão & 990 & 1,00 & $0,88-1,00$ \\
\hline Traumatismo intra-abdominal & 984 & 0,93 & $0,87-0,99$ \\
\hline Queimaduras & 990 & 1,00 & $0,84-1,00$ \\
\hline Intoxicações & 989 & 0,98 & $0,89-1,00$ \\
\hline Complicações de cuidados médicos & 970 & 0,82 & $0,75-0,90$ \\
\hline Seqüelas de lesões & 979 & - & - \\
\hline \multicolumn{4}{|l|}{ Causas externas } \\
\hline Acidentes de transporte & 594 & 0,90 & $0,88-0,93$ \\
\hline Pedestre & 150 & 0,84 & $0,78-0,90$ \\
\hline Ciclista & 100 & 0,80 & $0,72-0,88$ \\
\hline Motociclista & 142 & 0,84 & $0,78-0,90$ \\
\hline Ocupante de automóvel e caminhonete & 92 & 0,65 & $0,55-0,74$ \\
\hline Quedas & 452 & 0,83 & $0,80-0,87$ \\
\hline Queda não especificada & 232 & 0,68 & $0,62-0,75$ \\
\hline Demais causas acidentais & 88 & 0,49 & $0,39-0,59$ \\
\hline Agressões & 72 & 0,51 & $0,40-0,62$ \\
\hline Lesões autoprovocadas intencionalmente & 16 & 0,72 & $0,51-0,94$ \\
\hline Complicações da assistência médica & 2 & 0,03 & $-0,03-0,09$ \\
\hline Causas indeterminadas & 214 & 0,37 & $0,30-0,44$ \\
\hline Eventos e intenção indeterminados & 204 & 0,49 & $0,43-0,56$ \\
\hline Seqüelas de causas externas & 980 & - & - \\
\hline
\end{tabular}


pesquisador estão apresentadas na Tabela 3 . A concordância entre os dados registrados na AIH e na avaliação do prontuário com o coeficiente Kappa foi considerada "ótima" para quase todos os grandes grupos de lesões, exceto para o segmento corporal: "traumatismos do pescoço" (0,73-boa) e "traumatismos múltiplos" (0,67-boa); nas naturezas da lesão: "fratura do pescoço" (0,72-boa) e "fratura do tórax" (0,49-regular) (Tabela 3).

O grau de concordância nos tipos de causas externas foi, de um modo geral, menor do que nas lesões (Tabela 3). Concordância ótima: "quedas" $(0,83)$, "acidentes de transporte" $(0,90)$, "pedestre" $(0,84)$ e "motociclista" $(0,84)$. Concordância boa: "ciclista" $(0,80)$, "ocupante de automóvel e caminhonete" $(0,65)$, "queda não especificada" $(0,68)$ e lesões autoprovocadas $(0,72)$. Concordância regular: "demais causas acidentais" $(0,49)$, "agressões" $(0,51)$, e "eventos e intenção indeterminados" $(0,49)$. Concordância sofrível: "causas indeterminadas" $(0,37)$ e concordância fraca: "complicações da assistência médica” $(0,03)$.
A Tabela 4 mostra uma comparação da morbidade hospitalar proporcional por causa externas no SIH-SUS e na avaliação do pesquisador. Os acidentes de transporte e as quedas foram respectivamente, a primeira e segunda causa de internação em ambas as avaliações, com valores próximos. No SIH-SUS, os acidentes de transporte representaram $31,8 \%$ e as quedas $25,8 \%$. Na avaliação do pesquisador, respectivamente, $32,3 \%$ e $26,3 \%$.

Houve um acréscimo de 5 internações por acidentes de transporte na avaliação do pesquisador, porém a concordância ocorreu em 297 casos. Foram recuperadas dos prontuários, como acidentes de transporte, 23 internações por outros tipos de causas externas e foram consideradas como acidentes de transporte, no SIH-SUS, 18 internações por outros tipos de causas externas. Em relação às quedas houve um acréscimo de 5 internações com concordância em 226. A avaliação do prontuário recuperou, como quedas, 34 internações sendo 27 delas consideradas no SIH-SUS como causas indeterminadas. Também no

Tabela 4 - Comparação de tipo de causa externa na SIH e na avaliação do pesquisador, de internações no Hospital Municipal de São José dos Campos, primeiro semestre de 2003.

Table 4 - Comparison of the type of external-cause in the SIH and in the researcher's evaluation of admissions in the Municipal Hospital of São José dos Campos, first semester of 2003.

\begin{tabular}{|c|c|c|c|c|c|c|c|c|c|c|c|c|}
\hline \multirow{2}{*}{ Avaliação do Pesquisador } & \multicolumn{10}{|c|}{$\mathrm{SIH}$} & \multicolumn{2}{|c|}{ Total } \\
\hline & 1 & 2 & 3 & 4 & 5 & 6 & 7 & 8 & 9 & 10 & $\mathrm{~N}$. & $\%$ \\
\hline 1. Acidentes de transporte & 297 & 7 & 2 & 1 & - & - & 13 & - & - & - & 320 & 32,3 \\
\hline 2.Quedas & 4 & 226 & 3 & - & - & - & 27 & - & - & - & 260 & 26,3 \\
\hline 3.Demais causas acidentais & 3 & 10 & 44 & 3 & 1 & - & 20 & - & - & - & 81 & 8,2 \\
\hline 4.Agressões & - & - & 10 & 36 & - & - & 3 & - & - & - & 49 & 4,9 \\
\hline $\begin{array}{l}\text { 5. Lesões autoprovocadas } \\
\text { intencionalmente }\end{array}$ & - & - & 3 & - & 8 & - & 1 & - & - & - & 12 & 1,2 \\
\hline $\begin{array}{l}\text { 6. Complicações da assistência } \\
\text { médica }\end{array}$ & 4 & 1 & 1 & - & - & 1 & 53 & - & - & - & 60 & 6,1 \\
\hline 7. Causas indeterminadas & 3 & 10 & 20 & 43 & 1 & - & 107 & - & - & - & 184 & 18,6 \\
\hline 8. Seqüelas & 4 & - & - & 1 & - & - & 5 & - & - & - & 10 & 1,0 \\
\hline 9. Causas naturais & - & 1 & - & - & - & - & 4 & - & - & - & 5 & 0,5 \\
\hline 10. Informação insuficiente & - & - & 1 & - & - & - & 8 & - & - & - & 9 & 0,9 \\
\hline Total (n) & 315 & 255 & 84 & 84 & 10 & 1 & 241 & - & - & - & 990 & \\
\hline Total (\%) & 31,8 & 25,8 & 8,5 & 8,5 & 1,0 & 0,1 & 24,3 & - & - & - & & 100,0 \\
\hline
\end{tabular}


SIH-SUS foram codificadas como quedas, 29 internações por outras causas externas. Das AIHs codificadas como causas externas, 5 foram descartadas pelo pesquisador e 9 foram consideradas com informação insuficiente para caracterizar, se foi uma internação por causa externa.

Em relação às agressões o perfil da morbidade foi bastante modificado. Caiu de $8,5 \%$ no SIH-SUS para 4,9\% na avaliação do pesquisador (Tabela 4). Também as complicações da assistência médica foram bastante alteradas na comparação. De um caso $(0,1 \%)$ no SIH, subiu para 60 casos $(6,1 \%)$. Embora a concordância para complicações de cuidados médicos no diagnóstico principal tenha sido boa (Tabela 3) não houve uma correspondente qualidade de codificação de causa externa no diagnóstico secundário. Regularmente esses casos foram codificados, erroneamente no SIH, como "evento cuja intenção é indeterminada".

\section{Discussão e conclusão}

Deve-se destacar o fato de que as internações pagas pelo SUS não representam a totalidade dos casos, visto que os usuários de seguro-saúde e particulares não estão incorporados nesse sistema de informação. No entanto, em relação às causas externas, é possível afirmar grande parte dos atendimentos acabam sendo direcionados primeiramente para os hospitais públicos ou conveniados do SUS, devido ao caráter de emergência do atendimento.

Uma limitação do estudo foi a exclusão das internações codificadas no SIH-SUS como sendo causas naturais, para avaliação da qualidade dos dados. Portanto, aquelas AIHs codificadas como causas naturais, mas que na verdade eram causas externas, não puderam ser recuperadas e incluídas nas análises de concordância.

A análise dos dados restringiu-se a somente um hospital do Município, embora no mesmo tivessem ocorrido $92,3 \%$ das internações por lesões decorrentes de causas externas no período de estudo, como já foi referido. Portanto, as conclusões sobre a qualidade dos dados foram aplicáveis somente a este hospital.

No Município de São José dos Campos, SP, no final de 1997, foi realizado um treinamento para os coletores de dados da AIH sobre morbidade em CID-10, com o objetivo de aperfeiçoar o registro dos diagnósticos de internação no SIH-SUS ${ }^{19}$. A codificação de doenças foi feita por profissionais administrativos no setor de faturamento, onde os procedimentos hospitalares eram codificados. A única alternativa possível foi treinar esses "faturistas" para codificação de morbidade, segundo as regras da CID10 , orientando-se os mesmos à pesquisa da lesão (diagnóstico principal) no resumo de alta, e da causa externa (diagnóstico secundário) na ficha de atendimento de emergência ou na ficha de evolução clínica do paciente. A pesquisa ora realizada não teve o escopo de avaliar esse processo de trabalho. Porém, é possível que, devido à estabilidade da equipe de faturistas no período, a qualidade dos dados referentes ao diagnóstico secundário de internação tenha sido influenciada por essa metodologia.

A prática encontrada de digitação do código IBGE de São José dos Campos, mesmo quando o paciente residia em outro município ( $8,7 \%$ dos casos) é potencialmente prejudicial em duas diferentes formas de utilização das informações do SIH-SUS. A primeira é para o cálculo da taxa de internação por local de residência. Os dados serão superestimados para São José dos Campos. A segunda é para os pactos de regionalização da assistência hospitalar. Nesse caso a proporção de internação de pacientes de outros municípios será subestimada.

Uma explicação para a boa qualidade dos dados relativos à vítima e à internação está na importância de sua exatidão para o correto faturamento das AIHs. Também em relação às lesões (diagnóstico principal), foi encontrada uma característica diferenciada de codificação que provavelmente contribuiu para essa concordância elevada das lesões, que foram as regras de codificação de morbidade por causa única, que elege o procedimento mais importante realizado 
como o diagnóstico principal.

Em 2003, já vigorava a “Tabela de Crítica de Compatibilidade Procedimento x CID10 "25. Esta tabela impõe uma relação de códigos em CID-10 compatíveis para cada procedimento. O objetivo dessa tabela é evitar que sejam registrados nos bancos de dados do SIH-SUS códigos de diagnóstico principal incompatíveis com os procedimentos realizados.

Esta característica influenciou negativamente a taxa de concordância bruta da codificação no nível de subcategoria de quatro caracteres do diagnóstico principal. A avaliação do pesquisador não levou em consideração essa tabela, mas considerou o procedimento realizado para sua decisão de codificação. Isto explica a baixa taxa de concordância bruta. A maioria das diferenças de critérios de compatibilidade se deu nesse nível de classificação. Do total de codificações discordantes, $15,2 \%$ podem ter ocorrido porque a tabela não permitia o código selecionado pelo pesquisador. No entanto, a discordância potencialmente provocada pela tabela foi bem menos importante em nível de categorias de três caracteres $(4,1 \%)$ e de agrupamento $(3,9 \%)$ da CID-10, que são as classificações mais freqüentemente utilizadas nas tabulações estatísticas. No entanto, considera-se que as discordâncias elevadas no nível de quatro caracteres da CID são de pouca validade para estudos epidemiológicos ${ }^{4}$.

Já em relação às causas externas (diagnóstico secundário), dois fatores tiveram importância na confiabilidade dos dados. O primeiro é que inexiste um local específico e padronizado para colocação da causa externa no prontuário. Vários ou nenhum registro podiam ser encontrados nos diversos impressos que compõem o prontuário do paciente. Isto prejudicou a localização da informação mais adequada, dificultando a decisão do observador. Outro fator foi a pouca preocupação dos médicos em registrar a causa da lesão. Era comum encontrar registros do tipo: "FAF" (ferimento por arma de fogo), "FAB" (ferimento por arma branca), "acidente de trânsito", "trauma de joelho", etc. Freqüentemente, a informação era mais detalhada na ficha de enfermagem, principalmente para acidentes de transporte.

Embora a compreensão da letra dos médicos fosse difícil em algumas situações, foi possível entender a escrita em todos os casos e supõe-se que os codificadores do hospital tivessem também essa capacidade de compreensão, dada a grande familiaridade dos mesmos com o manuseio de prontuários e também pelo fato de terem sido treinados. Na opinião do pesquisador, a maior dificuldade dos codificadores foi a necessidade de vasculhar o prontuário em busca de informações sobre causa externa, pois, como já foi salientado, inexiste um local apropriado para o seu registro.

Os agrupamentos utilizados para a análise de concordância dos diagnósticos principal e secundário foram muito diversificados, por necessidade do estudo. Por conseguinte, muitos resultados apresentaram baixa prevalência e a proporção de verdadeiros negativos foi muito alta. Segundo Pereira 22: "Baixas prevalências tendem a estar associadas a baixos níveis de reprodutibilidade, pois o valor de Kappa depende da concordância devida ao acaso". Essa seria uma explicação para os valores encontrados em "traumatismo do pescoço", "fratura do pescoço", "fratura do tórax", "lesões autoprovocadas" e "complicações da assistência médica”.

A redução da proporção de agressões na avaliação do pesquisador em relação ao encontrado no SIH-SUS foi provocada pela codificação equivocada de agressão quando não havia informação no prontuário sobre a intencionalidade do ato, principalmente por arma de fogo e por objeto cortante. Embora houvesse a grande probabilidade de se tratar de agressão, a regra de codificação não permite tal dedução sem o registro desse fato em prontuário.

Deve-se destacar que a proporção de internações pagas pelo SUS por causas externas no primeiro semestre de 2003, com as AIHs de todos os hospitais do Município foi de $28,8 \%$ para acidentes de transporte e $26,3 \%$ para quedas ${ }^{18}$. Esses valores foram 
muito próximos aos resultados obtidos no presente estudo, realizado somente no Hospital Municipal de São José dos Campos, o que dá uma noção de que a distribuição proporcional das internações por causas externas é semelhante no referido hospital e no Município.

Poucos estudos avaliaram a qualidade dos dados de morbidade por causas externas no Brasil. Mesquita Filho ${ }^{26}$ estudou 380 vítimas de causas externas atendidas em serviço de pronto-atendimento na cidade de Pouso Alegre, MG, em 2003. Os resultados para grupos de lesões e causas externas mostraram: concordância perfeita para acidente de transporte; ótima para trauma de cabeça, de membros superiores e de membros inferiores; sofrível para agressões. No presente estudo, a concordância foi ótima para acidentes de transporte, para trauma de cabeça, membros superiores e membros inferiores, para agressões, foi regular.

O estudo de Mathias e Soboll ${ }^{9}$ avaliou a qualidade do campo diagnóstico principal da AIH, em Maringá, PR, em 1992. Foram analisadas as concordâncias para dois agrupamentos de lesões: "fraturas", cujo coeficiente Kappa foi 0,79 e "lesões intracranianas e internas, incluindo os nervos", com Kappa de 0,82. No estudo de São José dos Campos, o coeficiente Kappa para fraturas foi 0,89 e para traumatismo intracraniano, 0,95.

Quanto à confiabilidade dos dados sobre qualidade da vítima de acidente de transporte, um estudo realizado sobre esse tema em Londrina, em 1996, por Andrade e Mello Jorge ${ }^{11}$, teve modificado o perfil original e perfil pós-investigação. Houve um aumento em todos os grupos. Em pedestres foi de $+29,8 \%$, em ciclistas de $+216,7 \%$, em motociclistas de $+131,6 \%$ e em ocupantes de automóvel e caminhonete $+32,6 \%$. No caso de Londrina a maior migração se deu a partir do agrupamento das "causas indeterminadas”; em São José dos Campos, a maior migração ocorreu dentro do próprio agrupamento dos acidentes de transporte, a partir do grupo "ocupantes de automóvel e caminhonete".
A ocorrência de praticamente uma internação por acidente de transporte para cada internação por queda, com ou sem correção dos dados da AIH, contrariou a tendência geral da morbidade hospitalar por causas externas relatada por outros autores, onde a principal causa destacada de internações por essa causa é representada pelas quedas. Gawryszewski e col. ${ }^{27}$ estudaram as internações por causas externas pelo SUS no Brasil em 2000 e identificaram $42,8 \%$ de internações por quedas e $18,2 \%$ de internações por acidentes de transporte.

No Estado de São Paulo, em 2003, dos dez municípios paulistas com mais de 400.000 habitantes, somente São José dos Campos teve os acidentes de transporte como principal causa de internação por causas externas. A razão de internação entre acidentes de transporte e quedas nesses municípios variou de 3,9:1 até 1,8:1 ${ }^{18}$.

Considerando-se que a confiabilidade dos dados sobre internações sobre acidentes de transporte e por quedas em São José dos Campos foi boa, levanta-se a questão de que o Município tem realmente um importante problema com a violência no trânsito, condição já sugerida por Melione ${ }^{28}$. Também fica a indagação se existem problemas com a qualidade da codificação do diagnóstico secundário por causas externas em outros municípios, principalmente em relação ao grupo "quedas". O resultado da pesquisa realizada por Tomimatsu ${ }^{12}$, em Londrina, em 2004, sugere essa possibilidade, já que após correção dos dados do SIHSUS mediante consulta ao prontuário dos pacientes e outros documentos, o perfil da morbidade hospitalar por causas externas foi modificado de 56,7\% internações por quedas e $16,4 \%$ por acidentes de transporte, para $20,3 \%$ e $24,6 \%$, respectivamente.

Face à relevância das informações obtidas neste estudo, tanto do banco de dados do SIH-SUS como dos dados avaliados pelo pesquisador, recomenda-se o investimento das esferas municipal, estadual e federal do SUS para o aperfeiçoamento da qualidade dos dados epidemiológicos, nesse sistema de informação. Esse tipo de informação é 
importante para a análise da situação de saúde e de outros fenômenos atuais da nossa sociedade que interferem no setor saúde, como a violência urbana.

Até a realização desta pesquisa, inexistiam, no Município de São José dos Campos, estudos que aprofundassem a avaliação da qualidade dos dados de internação hospitalar causas externas. Esta necessidade era premente para se fazer um diagnóstico de saúde que incluísse os dados de morbidade para complementar as análises de mortalidade, nos tempos em que fatores relacionados à assistência médica, à dinâmica social e às políticas públicas para o controle dos acidentes e a violência, influenciam drasticamente no perfil da mortalidade.

Após a conclusão do estudo, foram encaminhadas sugestões à Secretaria de Saúde da Prefeitura Municipal de São José dos Campos, visando a melhoria da qualidade da codificação das variáveis relativas à vítima, à internação e ao agravo que apresentaram problemas com a qualidade dos dados. O acesso dos codificadores de $\mathrm{AIH}$ às fichas de atendimento pré-hospitalar, de notificação de violência e da Declaração de Óbito contribuiria para a melhor qualidade dos dados sobre internações por causas externas.

\section{Referências}

1. Mello Jorge MHP, Koizumi MS. Gastos governamentais do SUS com internações hospitalares por causas externas: análise no Estado de São Paulo, 2000. Rev Bras Epidemiol 2004; 7(2): 228-38.

2. Drumond Jr M, Lira MMTA, Freitas M de, Nitrini TMV, Shibao K. Avaliação da qualidade das informações de mortalidade por acidentes não especificados e eventos com intenção indeterminada. Rev Saúde Pública 1999; 33(3): 273-80.

3. [OMS] Organização Mundial da Saúde. Manual de vigilância das lesões. São Paulo: Secretaria de Estado da Saúde de São Paulo; 2004.

4. Lebrão ML. Estudos de morbidade. São Paulo: USP; 1997. Estatísticas hospitalares; p. 59-72.

5. Carvalho DM. Grandes sistemas nacionais de informação em saúde: revisão e discussão da situação atual. Inf Epidemiol SUS 1997; 6(4): 7-46.

6. Laurenti R, Mello Jorge MHP, Gotlieb SLD. A confiabilidade dos dados de mortalidade e morbidade por doenças crônicas não-transmissíveis. Ciênc Saúde Coletiva 2004; 9(4): 909-20.

7. Lebrão ML. Análise da fidedignidade dos dados estatísticos hospitalares disponíveis na Secretaria de Estado da Saúde de São Paulo em 1974. Rev Saúde Pública 1978; 12(2): 234-49.

8. Veras CMT, Martins MS. A confiabilidade dos dados nos formulários de autorização de internação hospitalar (AIH), Rio de Janeiro, Brasil. Cad Saúde Pública 1994; 10(3): 339-55.

9. Mathias TAF, Soboll MLMS. Confiabilidade de diagnósticos nos formulários de autorização de internação hospitalar. Rev Saúde Pública 1998; 32(6): 26-32.
10. Escosteguy CC, Portela MC, Medronho RA, Vasconcellos MTL. O sistema de informações hospitalares e a assistência ao infarto agudo do miocárdio. Rev Saúde Pública 2002; 36(4): 491-9.

11. Andrade SM, Mello Jorge MHP. Acidentes de transporte terrestre em cidade da Região Sul do Brasil: avaliação da cobertura e qualidade dos dados. Cad Saúde Pública 2001; 17(6): 1449-56.

12. Tomimatsu MFAI. Internações por acidentes e violências financiadas pelo setor público em Londrina, Paraná: análise dos registros, gastos e causas [dissertação de mestrado]. Universidade Estadual de Londrina: Paraná; 2006.

13. [OMS] Organização Mundial da Saúde. Classificação estatística internacional de doenças e problemas relacionados à saúde - $10^{a}$ revisão. São Paulo: Edusp; 1995.

14. Ministério da Saúde. Portaria n. 1311, de 12 de setembro de 1997. Define a competência janeiro de 1998, para que a CID-10 vigore, em todo o território nacional, em morbidade hospitalar e ambulatorial. Diário Oficial da União, Brasília, n. 178, p. 20.518, 16 set. 1997a. Seção 1.

15. Ministério da Saúde. Portaria n. 142, de 13 de novembro de 1997. Dispõe sobre o preenchimento de Autorização de Internação Hospitalar - AIH, em casos com quadro compatível com causas externas. Diário Oficial da União, Brasília, n. 222, p. 26.499, 17 nov. 1997b. Seção 1.

16. Ministério da Saúde. Secretaria Executiva. Departamento de Informática do SUS. Informações de Saúde. Demográficas e socioeconômicas. Censos (1980, 1991 e 2000), contagem (1996) e projeções intercensitárias (1981 a 2006), segundo faixa etária, sexo e situação de domicílio. Disponível em:http://tabnet. datasus.gov.br/cgi/deftohtm.exe?ibge/cnv/popsp.def (2006 mar 10) 
17. Moreira ML. Cobertura e utilização de serviços de saúde suplementar no Estado de São Paulo [dissertação de mestrado]. Escola Nacional de Saúde Pública da Fundação Osvaldo Cruz: Rio de Janeiro; 2004.

18. Ministério da Saúde. Secretaria Executiva. Departamento de Informática do SUS. Movimento mensal de internação hospitalar. [MS-BBS base de dados on-line]. Disponível em: http://msbbs.datasus.gov.br/ public/default.htm, [Acessado em 3 de abril de 2006].

19. Melione LPR. Utilização de informações hospitalares do sistema único de saúde para vigilância epidemiológica e avaliação de serviços ambulatoriais em São José dos Campos - São Paulo. Inf Epidemiol SUS 2002; 11(4): 21525.

20. Fleiss JL. Statistics methods for rates and proportions. New York: John Wiley \& Sons; 1981.

21. Landis Jr, Koch GG. The measurement of observer agreement for categorical data. Biometrics 1977; 33: 159174.

22. Pereira MG. Epidemiologia: teoria e prática. Rio de Janeiro: Guanabara Koogan; 1995.

23. Dean AG et al. EPI INFO. A word processing database, and statistics program for epidemiology on microcomputers. [programa de computador]. Version 6. Atlanta: Centers for Disease Control and Prevention; 1994.
24. Abramson JH, Gahlinger PM. PEPI. Computer programs for epidemiologists. Version 4.0. [programa de computador]. Salt Lake City: Sagebrush Press; 2001.

25. Ministério da Saúde. Portaria n. 579, de 20 de dezembro de 2001. Aprova a Tabela de Compatibilidade entre o Procedimento Realizado e o Diagnóstico Principal informado, de acordo com a CID-10 no processamento das AIHs do Sistema de Informações Hospitalares. Diário Oficial da União, Brasília, n. 244, p. 110, 26 dez. 2001b. Seção 1.

26. Mesquita Filho M. Vítimas de causas externas atendidas em serviço de urgência e emergência - subsídios ao desenvolvimento de sistema de informações. [tese de doutorado]. Faculdade de Saúde Pública da USP: São Paulo; 2003.

27. Gawryszewski VP, Koizumi MS, Mello Jorge MHP. As causas externas no Brasil no ano 2000: comparando a mortalidade e a morbidade. Cad Saúde Pública 2004; 20(4): 995-1003.

28. Melione LPR. Morbidade hospitalar e mortalidade por acidentes de transporte em São José dos Campos, São Paulo. Rev Bras Epidemiol 2004; 7(4): 461-72.

Recebido em: 04/06/07

Versão final reapresentada em: 08/05/08

Aprovado em: 25/06/08 